\title{
Elternzentrierte ethische Entscheidungsfindung für Frühgeborene im Grenzbereich der Lebensfähigkeit - Reflexion über die Bedeutung probabilistischer Prognosen als Entscheidungsgrundlage
}

\author{
André Kidszun
}

Eingegangen: 25. Januar 2021 / Angenommen: 5. Juli 2021 / Online publiziert: 18. August 2021 (C) Der/die Autor(en) 2021

Zusammenfassung Frühgeborene im Grenzbereich der Lebensfähigkeit befinden sich in einer prognostischen Grauzone. Das bedeutet, dass deren Prognose zwar schlecht, aber nicht hoffnungslos ist, woraus folgt, dass nach Geburt lebenserhaltende Behandlungen nicht obligatorisch sind. Die Entscheidung für oder gegen lebenserhaltende Maßnahmen ist wertbeladen und für alle Beteiligten enorm herausfordernd. Sie sollte eine zwischen Eltern und Ärzt*innen geteilte Entscheidung sein, wobei sie unbedingt mit den Präferenzen der Eltern abgestimmt sein sollte. Bei der pränatalen Beratung der Eltern legen die behandelnden Ärzt*innen üblicherweise numerische Schätzungen der Prognose vor und nehmen in der Regel an, dass die Eltern ihre Behandlungspräferenzen davon ableiten. Inwieweit probabilistische Daten die Entscheidungen der Eltern in prognostischen Grauzonen tatsächlich beeinflussen, ist noch unzureichend untersucht. In der hier vorliegenden Arbeit wird eine Studie reflektiert, in welcher die Hypothese geprüft wurde, dass numerisch bessere oder schlechtere kindliche Prognosen die Präferenzen werdender Mütter für lebenserhaltende Maßnahmen nicht beeinflussen. In dieser Studie zeigte sich, dass die elterlichen Behandlungspräferenzen eher von individuellen Einstellungen und Werten als von Überlegungen zu numerischen Ergebnisschätzungen herzurühren scheinen. Unser Verständnis, welche Informationen werdende Eltern, die mit einer extremen Frühgeburt konfrontiert sind, wünschen und brauchen, ist noch immer unvollständig. Bedeutende medizinische Entscheidungen werden keineswegs nur rational und prognoseorientiert gefällt. In der vorliegenden Arbeit wird diskutiert, welchen Einfluss der Prozess der Entscheidungsfindung auf das Beratungsergebnis haben kann und welche Implikationen sich aus den bisher vorliegenden Studienergebnissen ergeben - klinisch-praktisch, ethisch und wissenschaftlich.

PD Dr. med. André Kidszun, MA $(\bowtie)$

Abteilung für Neonatologie, Universitätsklinik für Kinderheilkunde, Universitätsspital Bern,

Universität Bern, Freiburgstraße 15, 3010 Bern, Schweiz

E-Mail: andre.kidszun@insel.ch 
Schlüsselwörter Entscheidungsfindung · Prognose · Grenzbereich der

Lebensfähigkeit · Frühgeburt · Probabilistische Daten

\title{
Parent-centered ethical decision making for preterm infants born near the limit of viability — reflection on the significance of probabilistic prognoses as a basis for shared decision making
}

\begin{abstract}
Definition of the problem Decision making for preterm infants born near the limit of viability is complex, value-laden, and challenging for parents as well as healthcare professionals. When counseling parents, healthcare professionals usually present numerical estimates of prognoses (e.g., \% probability of survival) and often assume that parents will derive their treatment preferences from such prognostic information. It is, however, unclear whether probabilistic data actually influence parents' preferences in prognostic gray zones, where prognosis is generally poor and lifesustaining treatments are not mandatory.

Arguments Our understanding of what information parents facing extremely preterm birth want and need remains incomplete and requires further studies. Dramatic and far-reaching medical decisions are by no means made only rationally and prognosis oriented. In this essay, the role of probabilistic prognoses during parent-centered decision making in prognostic gray zones is evaluated on the basis of the available evidence. It will be argued that parental treatment preferences rather originate from individual attitudes and values than from considerations of numerical outcome estimates.
\end{abstract}

Summary Conceptual approaches referring to personalized decision making, and relational autonomy will be presented and discussed.

Keywords Parents $\cdot$ Decision making $\cdot$ Prognosis $\cdot$ Limit of viability $\cdot$ Preterm birth $\cdot$ Probabilistic data

\section{Einleitung}

Entscheidungen für Frühgeborene im Grenzbereich der Lebensfähigkeit und damit über den Einsatz oder die Begrenzung lebenserhaltender Maßnahmen, sind ein essentielles Behandlungselement der modernen Neonatologie. Sowohl das Fachgebiet als auch die spezifischen Probleme der Entscheidungsfindung haben sich in den vergangenen 50 Jahren auf entscheidende Weise weiterentwickelt. Noch in den 1970erJahren waren die therapeutischen Möglichkeiten begrenzt und Kinder mit einem Gestationsalter von weniger als 29 Wochen galten generell als nicht überlebensfähig (Carlsson und Svenningsen 1975). In der zweiten Hälfte des 20. Jahrhunderts begann jedoch die intensivmedizinische Behandlung von Frühgeborenen enorme Fortschritte zu machen. In den letzten Jahrzehnten hat der Einsatz potentiell lebensverlängernder medizinischer Maßnahmen bei sehr unreifen Frühgeborenen nicht nur zugenommen, (Versluys und de Leeuw 1995) mit dem Einsatz künstlicher Beatmung, medikamentöser Unterstützung des Blutkreislaufs, parenteraler Ernährung 
und anderen Maßnahmen gelingt es heutzutage sehr viele dieser sehr unreifen Kinder am Leben zu halten, und auch gesund nach Hause zu entlassen. In einigen Fällen sterben Kinder jedoch trotz der medizinischen Maßnahmen oder sie überleben, ohne dass ihnen eine zumindest minimale Lebensqualität erhalten bleibt. Der Einsatz intensivmedizinischer Maßnahmen ist somit keinesfalls immer benefitär im Sinne des Kindeswohls, sondern kann auch Leidensverlängerung, Herauszögern des Todes oder ein Überleben mit schwersten neurologischen Schäden bedeuten (American Academy of Pediatrics Committee on Fetus and Newborn und Bell 2007).

1973 wurde erstmals über die neu erwachsenden moralischen und ethischen Probleme der modernen neonatologischen Intensivmedizin berichtet (Duff und Campbell 1973). Raymond Duff und Alexander Campbell beschrieben darin eine Gruppe von Kindern, deren Prognose so schlecht eingeschätzt wurde, dass keine Aussicht auf ein ,sinnhaftes (meaningful)“ Leben bestand und daher auf ,weitere“ Therapie verzichtet wurde. Diese Entwicklung zur bewussten Begrenzung von intensivmedizinischen Therapien hat sich seither fortgesetzt. Die Auswertungen mehrerer großer populationsbasierter Studien zeigen, dass wenn heutzutage ein Kind im Bereich der neonatologischen Intensivmedizin verstirbt, dem in aller Regel eine Entscheidung zur Therapiebegrenzung vorausgeht (van der Heide et al. 1997; Costeloe et al. 2000; Larroque et al. 2004; Hentschel et al. 2006). Der Einsatz und die Begrenzung lebenserhaltender Maßnahmen bei Frühgeborenen im Grenzbereich der Lebensfähigkeit ist oft ethisch problematisch und trotz der enormen Tragweite der Entscheidungen sind nur wenige klare ethisch-praktische Orientierungspunkte verfügbar. Auf welcher Grundlage soll entschieden werden, was am besten zu tun ist?

Ziel der vorliegenden Arbeit ist es, die Grundlagen und Einflussfaktoren für solche Entscheidungen näher zu charakterisieren. Nach einer einführenden Darstellung der allgemeinen Probleme bei der Entscheidungsfindung auf Grundlage zahlenmäBiger Prognosen werden die Ergebnisse einer empirischen Studie vorgestellt und reflektiert. Mit Fokus auf die elterlichen Bedürfnisse im Entscheidungsprozess, werden abschließend Denkansätze entwickelt, welche als Grundlage für ein praktikables und ethisch verantwortliches Entscheidungsgerüst dienen könnten.

\section{Probleme bei der Präsentation und Interpretation medizinischer Fakten im Kontext von Behandlungsentscheidungen an der Grenze zur Lebensfähigkeit}

\section{Medizinische Fakten als Basis der Entscheidungsfindung}

Aus klinisch-praktischer Sicht ist die wesentliche Säule guter Entscheidungsfindung die Kenntnis und die korrekte Darstellung medizinischer Fakten. In diesem Zusammenhang sind sogenannte Entscheidungshilfen gut untersucht. Medizinische Entscheidungshilfen sind geeignet, um Betroffene besser zu informieren, Risiken und Nutzen von Behandlungsoptionen klarer zu machen, sie aktiver in den Entscheidungsprozess einzubinden und vermutlich auch, um Entscheidungen besser an deren individuellen Werten zu orientieren (Stacey et al. 2017). In vielen Bereichen 
der Medizin sind solche Entscheidungshilfen jedoch nicht ausreichend verfügbar (Lenz et al. 2012).

Im Prozess der gemeinsamen Entscheidungsfindung werden zunehmend auch in der Neonatologie, meist visuelle, Entscheidungshilfen eingesetzt (Mactier et al. 2020). Solche Entscheidungshilfen sind für Eltern ungemein nützlich, und helfen prognostische Informationen besser $\mathrm{zu}$ erinnern und $\mathrm{zu}$ verstehen, scheinen deren Entscheidungskonflikt jedoch nicht zu beeinflussen (Guillén et al. 2012, 2019; Guillen und Kirpalani 2018). Eine Ursache könnte darin liegen, dass sich die Entscheidungshilfen auf die Darstellung populationsbasierter Prognosen fokussieren (Mactier et al. 2020) und die im Entscheidungsprozess zu ergründenden, individuellen elterlichen Werte zu wenig adressiert werden (Gaucher et al. 2016; Kukora und Boss 2018). Die im Entscheidungsprozess präsentierten Outcome Daten, insbesondere die Überlebensraten, sind jedoch nicht nur abhängig von der Art der Darstellung, sondern auch ganz wesentlich von deren Erhebung. Sie hängen nicht zuletzt von den (eigentlich zur Diskussion stehenden) prä-, peri- und postnatal durchgeführten medizinischen Maßnahmen ab. So schwanken beispielsweise die Überlebensraten von Frühgeborenen mit einem Gestationsalter von 23 Schwangerschaftswochen signifikant. Dies ist im Wesentlichen darauf zurückzuführen, dass der Anteil an Frühgeborenen, die bei diesem Gestationsalter lebenserhaltend behandelt werden, in verschiedenen Krankenhäusern eines Landes zwischen 0\% und 100\% schwankt (Rysavy et al. 2015). Mehrere Studien zeigen, dass vermehrter Einsatz lebenserhaltender Maßnahmen bei sehr unreifen Frühgeborenen zu einem verbesserten Gesamtüberleben ohne Erhöhung des Anteils der Kinder mit schweren neurokognitiven Einschränkungen führt (Håkansson et al. 2004; Serenius et al. 2016). Noch schwieriger als das Überleben lassen sich neurokognitive Defizite bestimmen und voraussagen. Bei der Beratung von Eltern mit drohender Frühgeburtlichkeit werden häufig dichotomisierte Ergebnisse dargestellt. Gewonnen werden solche Daten mit Hilfe entwicklungsneurologischer Tests (meist Bayley Scales of Infant Development) im korrigierten Alter von etwa 18 bis 24 Lebensmonaten. Anhand von spezifischen, numerischen Parametern (Motor Developmental Index (MDI)) wird mittels eines Cutoffs beispielsweise eine schwere von einer geringen Beeinträchtigung unterschieden. Neurokognitive Defizite sind jedoch individuell enorm unterschiedlich ausgeprägt und werden auch bezüglich ihrer Belastung als sehr unterschiedlich empfunden. Die kategorisierte Angabe von Beeinträchtigungen als mild oder schwer bildet teils nicht die realen Auswirkungen einer Behinderung ab. Der positive Vorhersagewert für ein signifikantes neurokognitives Defizit im Schulalter oder später ist zusätzlich sehr gering. Viele Kinder haben im Alter von zwei Jahren einen auffälligen Test, der sich später als nichtzutreffend herausstellt (Hack et al. 2005; Holsti et al. 2016). Die relativ frühe Testung überzeichnet demnach das Ausmaß der neurokognitiven Beeinträchtigungen. Insbesondere Outcome Statistiken müssen demnach sorgfältig analysiert und interpretiert werden. So berichten Studien über signifikant verschiedene Überlebensraten bei sehr unreifen Frühgeborenen. Dieser Umstand ist unter anderem dadurch bedingt, dass unterschiedliche Referenzpopulationen gewählt werden. So ist die Überlebensrate höher, wenn nicht alle, sondern nur Kinder betrachtet werden, die tatsächlich auf die Intensivstation aufgenommen wurden. Ähnliches gilt für die Darstellung von neurokognitiven Defiziten. 
Medizinische Fakten werden zudem aufgrund von Werthaltungen unterschiedlich interpretiert. Innerhalb Europas gibt es insgesamt deutliche Unterschiede in den nationalen und regionalen Handlungsempfehlungen (Cuttini et al. 2000). Ärzt*innen bewerten die Prognose von Frühgeborenen auch anders als Nicht-Professionelle. In einer Umfrage bewerteten $93 \%$ der befragten Neonatolog*innen eine intensivmedizinische Therapie bei 23 Schwangerschaftswochen oder darunter als ,aussichtslos“ (Peerzada et al. 2004). Wenn hingegen Erwachsene ohne eigene Vorerfahrungen mit der Prognose eines solchen Kindes bei 23 Schwangerschaftswochen konfrontiert werden, dann geben im Gegensatz etwa drei Viertel an, das Kind aktiv lebenserhaltend versorgen zu wollen (Haward et al. 2008).

Medizinische Fakten allein, ohne in einen lebensweltlichen Kontext gebracht und diskutiert worden zu sein, sind demnach nur bedingt als Grundlage der Entscheidungsfindung geeignet (Dionne-Odom und White 2021). Medizinische Fakten können Auskunft über das Outcome von ausgewählten untersuchten Populationen geben, beispielsweise wie viele Kinder, die in ähnlicher Situation geborenen wurden, überlebten, und welche Beeinträchtigungen bei den Überlebenden vorlagen. In medizinischen Graubereichen, in denen die Prognose sehr ungewiss, zugleich aber auch sehr schlecht ist, kann eine individuelle Prognose damit nur mit großen Schwierigkeiten abgeben werden.

\section{Bestimmung von Prognose und Behandlungsempfehlung}

Der Bestimmung der Prognose wird im Entscheidungsprozess dennoch eine zentrale Rolle beigemessen. Die Besonderheit medizinischer Prognosen in der Perinatologie liegt im Ausmaß der Unsicherheit über das zu erwartende Behandlungsergebnis. Es ist ungewiss, ob Komplikationen und Langzeitfolgen auftreten, welche Komplikationen und Langzeitfolgen auftreten und in welchem Ausmaß diese eintreten. Es besteht Einigkeit darüber, dass den Entscheidungsträgern, in der Regel den Eltern, im Rahmen der Beratung die Prognose bestmöglich und evidenzbasiert dargestellt bzw. vorausgesagt werden sollte. So wird in Leitlinien explizit auf die Wertigkeit von Prognoseaussichten eingegangen (Bührer et al. 2020). Zudem wurden, wie bereits erwähnt, Entscheidungshilfen entwickelt, damit Eltern besser verstehen, was Frühgeburt bedeutet und welche Folgen sie haben kann - jetzt aktuell und später im Leben (Mactier et al. 2020). Wichtig ist die gemeinsame Beratung und medizinische Betreuung durch die zwei involvierten Fachrichtungen - Geburtshilfe und Neonatologie, auch wenn dies in der Praxis noch zu wenig umgesetzt wird (Reed et al. 2020). Die Vorhersage von Prognosen kann nur individualisiert und anhand für die Eltern relevanter Parameter erfolgen. Man muss annehmen, dass Eltern verstehen wollen, wie die Prognose für ihr Kind und ihre Familie in ihrem speziellen Fall ist.

Zahlenmäßige Überlebensprognosen sind nicht nur schwierig zu beurteilen und den Eltern zu vermitteln, die Relevanz der zahlenmäßigen Prognosen hinsichtlich der Behandlungsentscheidung bzw. der Behandlungspräferenz der Eltern ist auch unzureichend evaluiert. Unterschiedliche Meinungen werden hinsichtlich der notwendigen Genauigkeit der Prognose vertreten. Die Frage, was eine 50\%ige Überlebenswahrscheinlichkeit für Eltern bedeutet, beantwortet die Neonatologin und Mutter Annie Janvier mit: „For parents, a 50\% risk of mortality does not mean much 
as their child will be either 100\% alive or 100\% dead" (Haward und Janvier 2015, S. 342). Möglicherweise sind relativ geringe Unterschiede in Überlebensprognosen (beispielsweise $10 \%$ oder $20 \%$ höher bzw. niedriger) in der Beratungssituation zu abstrakt und für die tatsächliche Entscheidungsfindung nicht relevant. Während also stratifizierte, populationsbasierte prognostische Daten für Ärzt*innen enorm relevant sind, da hiervon übergeordnete Beratungs- und Behandlungsansätze abhängig sind, scheinen sie für die individuell betroffene Familie weniger relevant zu sein, da die individuelle Prognose weiter schwer vorhersagbar und einzigartig bleibt.

\section{Akteur*innen der Entscheidungsfindung}

Traditionell verstehen sich die behandelnden Ärzt*innen in Fragen von Gesundheit und Krankheit als Vertreter und Fürsprecher für das Kind. Sie nehmen eine sogenannte Garantenstellung für das Kind ein. Grundsätzlich und gesetzlich verbrieft, obliegen jedoch alle Entscheidungen, die das Kind betreffen, den Eltern. Die meisten Ärzt*innen und Pflegenden in der Neonatologie befürworten auch die Einbindung der Eltern in Entscheidungen zur Therapiebegrenzung, interessanterweise meinen jedoch nur die wenigsten, dass die Eltern auch die Verantwortung für eine solche Entscheidung übernehmen sollten (McHaffie et al. 2001). Einschlägige Behandlungsempfehlungen führen explizit die Wichtigkeit einer von Behandlungsteam und Eltern gemeinsam getragenen Entscheidung, die Unsicherheit von Prognosen und auch die notwendigen ethischen Abwägungen im Entscheidungsprozess aus (Bührer et al. 2020). Vermutlich mit dem Ziel, die Behandlungsempfehlung praktikabel zu machen, wird der medizinische Graubereich, der prognostische Bereich, in dem die elterlichen Präferenz vollumfänglich bindend ist, für Deutschland anhand unsicherer prognostischen Marker (Gestationsalter, Schätzgewicht) relativ eng und mit arbiträren Grenzen festgelegt (Bührer et al. 2020). Dieser Ansatz wird von anderen Autoren kritisiert und international unterschiedlich gehandhabt (Cummings und Committee on Fetus and Newborn 2015; Lantos 2019). Festzuhalten bleibt, dass Ärztinnen und Ärzte sehr unterschiedliche und sich ändernde Ansichten darüber haben, welche kindliche Prognose ausreichend ist, um lebenserhaltende Maßnahmen nach einer frühen Frühgeburt zu rechtfertigen (Wilkinson et al. 2018; Di Stefano et al. 2021). Manchmal wird angeführt, Eltern befänden sich in einer Art Ausnahmesituation, die schwerwiegende und weitreichende Entscheidungen zu sehr erschwere, oder, dass Eltern die Bürde der Verantwortung dieser schweren Entscheidungen nicht tragen können oder wollen. Das ist richtig, bedeutet jedoch nicht, dass sie weniger am Entscheidungsprozess teilhaben sollten, sondern, dass sie mehr Unterstützung benötigen. Von enormer Bedeutung ist in diesem Zusammenhang, dass Eltern und ehemalige sehr unreife und kranke Frühgeborene ihre Situation häufig anders - und zwar signifikant besser - einschätzen als die behandelnden Ärzt*innen (Saigal et al. 1999, 2000). Die Entscheidungen zur Begrenzung lebenserhaltender Maßnahmen werden von Ärzt*innen in diesem Gebiet häufig als ,,medizinische“, faktische Entscheidungen verstanden, als Entscheidungen, die objektiv erscheinen. Die Rolle des Behandlungsteams im Prozess der Entscheidungsfindung ist noch unterbestimmt. In einer Umfrage unter US-amerikanischen Neonatolog*innen zeigte sich, dass eine Mehrheit (77\%) der Meinung ist, dass Entscheidungen zur Begrenzung lebenser- 
haltender Maßnahmen gemeinsam mit den Eltern getroffen werden sollten. Nur weniger als die Hälfte $(40 \%)$ gab an, dass dies auch so in der Praxis stattfindet. Die Mehrheit der beratenden Ärzt*innen verstand ihre primäre Rolle in der Vermittlung von Sachinformationen an die Eltern, nur ein Viertel der Ärzt*innen sahen ihre Hauptaufgabe darin, Eltern zu unterstützen, Nutzen und Gefahren unterschiedlicher Handlungsoptionen abzuwägen (Bastek et al. 2005). Zudem scheinen Eltern und Ärzt*innen prognostische Informationen unterschiedlich zu wichten. Während die Diskussion der Eltern thematisch breit angelegt ist und Überleben und Hoffnung eine große Rolle spielen, fokussieren Ärzt*innen häufig auf probabilistische Prognosen und das neurologische Outcome (Lemmon et al. 2019). Ebenso konnte festgestellt werden, dass unmittelbare medizinische Themen (Zustand der Mutter, Behandlung nach der Geburt) häufiger in der Beratung thematisiert wurden als ethische Fragen. So wurden Umgang und Erfahrung mit Tod und Sterben, die elterliche Auffassung, was ein ,gutes Leben“ ausmachte, oder allgemeine Fragen bezüglich Religion und Glauben vergleichsweise selten besprochen (Bastek et al. 2005). Eltern fühlen sich bezüglich medizinischer Informationen mithin gut beraten. Eltern möchten an den Entscheidungen teilhaben - eine aktivere Rolle spielen -, sie möchten jedoch diese Entscheidungen nicht gänzlich unabhängig treffen (Partridge et al. 2005). Auch wenn die empirische Datenbasis gering ist, so gibt es doch einige Hinweise, dass die elterliche Präferenz, lebenserhaltende Maßnahmen nicht zu initiieren oder zu beenden, nur wenig von der exakten zahlenmäßig zu erfassenden Prognose zu Mortalität und Morbidität abhängig gemacht wird. Vielmehr leiten Religion, Glaube und Hoffnung diese Entscheidungen (Boss et al. 2008; Kukora und Boss 2018). Eltern wollen zudem ungern selbst entscheiden, lebenserhaltende Maßnahmen nicht durchzuführen. Die meisten Eltern nehmen an, dass die Prognose für ihr Kind besser ist, als die ärztlich dargestellte Prognose vermuten lässt. Sehr schlechte Prognosen werden, im Gegensatz zu sehr guten Prognosen, oft unrealistisch optimistisch rezipiert (optimism bias), wobei die Ursachen dafür und die Bedeutung für den Entscheidungsprozess noch relativ unklar sind (Nayak et al. 2021). Ganz generell scheint die Art, wie die Prognose ärztlicherseits dargestellt wird, die Eltern stark zu beeinflussen. Etwa ein Drittel aller Eltern hatte aufgrund der ärztlich dargestellten Prognose dauerhaft Angst um das Leben ihres Kindes, auch nach Entlassung (Partridge et al. 2005). Es erscheint notwendig, medizinische Fakten in verständliche Outcomes zu ,übersetzen“, d.h. in Outcomes die Eltern verstehen und in ihre Lebenswelt einordnen können.

\section{Entscheidungsfindung unter prognostischer Ungewissheit}

Ungewissheit ist ein weiteres zentrales Element im Prozess der Entscheidungsfindung. Die Eltern, aber auch das medizinische Fachpersonal, erleben Ungewissheit und müssen unter dieser Ungewissheit Entscheidungen von enormer Tragweite treffen. Ungewissheit in diesem Sinne besteht immer dann, wenn ,Situationen mehrdeutig, komplex, unvorhersehbar oder probabilistisch sind, wenn nicht ausreichend oder nur widersprüchliches Wissen vorhanden ist und sich die Betroffenen mit ihrem eigenen oder dem allgemeinen Kenntnisstand unsicher fühlen" (Brashers 2001, S. 478). Merle Mishel, eine Pionierin der Erforschung des Ungewissheitsheitserle- 
bens von Patient*innen, hat es wie folgt definiert: „Uncertainty is the inability to determine the meaning of illness-related events, occurring when the decision maker is unable to assign definite value to objects or events, or is unable to predict outcomes accurately“ (Mishel 1990, S. 254). Ungewissheit beeinflusst das Erleben von Situationen in unterschiedlicher Art und Weise. Sie kann als Bedrohung oder Gefahr wahrgenommen werden, gerade in der akuten Phase einer Erkrankung (Jansen et al. 2006). Ungewissheit kann jedoch auch eine gewisse psychische Entlastung bewirken, vornehmlich dann, wenn die Prognose oder der Zustand des Erkrankten schlecht ist und sich aus Ungewissheit Hoffnung speist (Mishel 1990). Die Facetten der Ungewissheit umfassen mindestens medizinische Fakten, persönliche Faktoren (Erlebnisse und Erfahrungen, Krankheitserleben) sowie soziale Faktoren (Reaktion der Umwelt auf mögliche Entscheidungen, sozialkonformes Handeln, Rolle in der Gesellschaft), (Brashers et al. 2003; Martin et al. 2010). Im Kontext der Neonatologie kann Ungewissheit besonders prominent sein, da zusätzlich zu der persönlichen, informellen und sozialen Ungewissheit eine situativ-interpersonale Ungewissheit dazukommt. Entscheidungen im Grenzbereich der Lebensfähigkeit werden fast immer in den ersten Lebenstagen oder bereits vorgeburtlich diskutiert und vorgenommen. Dies bringt mit sich, dass sich Behandlungsteam und Eltern in der Regel fremd sind und sich, um diese Entscheidung zu erarbeiten, häufig das erste Mal im Leben gegenüberstehen. Die erstmalige Interaktion von Menschen ist häufig von Ungewissheit geprägt, dies kann kognitiven Stress verursachen und die Aufnahme und den Austausch von Informationen beeinflussen (Berger und Calabrese 1975). Der Entscheidungsprozess wird hiervon entscheidend geprägt. Ungewissheit ist kein Gefühl, sondern ein kognitiver Zustand, der nicht aufzulösen ist. Ungewissheit ist ein wesentliches Kennzeichen der Arzt-Patienten Beziehung (Begenau et al. 2010). Ungewissheit ist nicht uniform, sie entspringt aus den unterschiedlichsten Quellen des Krankheitserlebens (Komplexität der Behandlung, Prognose, Identitätsprobleme, soziale Interaktionen, finanzielle Probleme) und kann nicht mit Hilfe einer allgemeingültigen Strategie angegangen werden (Martin et al. 2010). Ungewissheit kann auch nicht einfach durch (zusätzliche) medizinische Fakten so reduziert werden, so dass sie keinen Einfluss mehr hat. Ganz im Gegenteil, medizinische Fakten können zusätzliche Ungewissheit erzeugen, wenn sie beispielsweise weitere Alternativen eröffnen (die ihrerseits wieder neu bewertet werden müssen) oder wenn sie von Patient*innen nicht präferierte Haltungen unterstützen (Brashers 2001). Ungewissheit führt zudem zu Coping-Strategien seitens der Patient*innen. Wesentlich ist hier das Vermeiden der Aufnahme und der kognitiven Verarbeitung wichtiger medizinischer Informationen zu nennen. Die Coping-Strategien umfassen unter anderem Vermeidung von Gesprächen, Ignorieren von Teilinformationen und Abwertung von Informationen bzw. der Quelle der Informationen (Mishel 1988, 1990; Barbour et al. 2012). 


\section{Auswirkungen von Outcome Schätzungen auf die Entscheidungspräferenzen von Müttern, die vor einer Frühgeburt stehen: Ergebnisse einer randomisierten klinischen Studie (Kidszun et al. 2020)}

Wie bisher dargestellt, befinden sich Frühgeborene im Grenzbereich der Lebensfähigkeit (in Deutschland häufig als Schwangerschaftsalter von $<24+0$ Wochen definiert) in einer prognostischen Grauzone (Cummings und Committee on Fetus and Newborn 2015; Bührer et al. 2020). Deren Prognose ist zwar schlecht, aber nicht hoffnungslos, woraus folgt, dass nach Geburt lebenserhaltende Behandlungen nicht obligatorisch sind. Die Entscheidung für oder gegen lebenserhaltende Maßnahmen ist wertbeladen und für alle Beteiligten enorm herausfordernd (Lantos 2018). Sie sollte eine zwischen Eltern und Ärzt*innen geteilte Entscheidung sein, wobei sie unbedingt mit den Präferenzen der Eltern abgestimmt sein sollte (Antommaria et al. 2015; Gaucher et al. 2016). Bei der pränatalen Beratung der Eltern legen die behandelnden Ärzt*innen üblicherweise numerische Schätzungen der Prognose vor (z. B. \% Überlebenswahrscheinlichkeit, \% Überleben ohne schwerwiegende Beeinträchtigungen) und nehmen möglicherweise an, dass Eltern ihre Behandlungspräferenzen davon ableiten (Guillén et al. 2012). Innerhalb des Graubereiches der Lebensfähigkeit existieren abhängig von Prognose und Gestationsalter enorme Behandlungsunterschiede. In einer großen populationsbasierten Studie zeigte sich beispielsweise, dass Frühgeborene mit einem Gestationsalter von 22+6 Schwangerschaftswochen (SSW) in etwa $50 \%$ der Fälle aktiv lebenserhaltend versorgt wurden, während Frühgeborene eine Gestationswoche später, bei $23+6 \mathrm{SSW}$, bereits in mehr als $80 \%$ der Fälle so behandelt wurden (Rysavy et al. 2015). Die Gründe hierfür können mannigfaltig sein. In der hier vorgestellten Studie (Kidszun et al. 2020) sollte geprüft werden, ob dieser tatsächlich beobachtbare Unterschied in den Behandlungsraten Ausdruck elterlicher Präferenzen sein könnte. Auf Grundlage bisher verfügbarer, gegenläufiger Evidenz, wurde die Hypothese aufgestellt, dass numerisch bessere oder schlechtere kindliche Prognosen die Präferenzen werdender Mütter für lebenserhaltende Maßnahmen nicht beeinflussen. Von Dezember 2017 bis Januar 2019 wurde eine doppelblinde, randomisierte Studie durchgeführt, in die werdende Mütter mit einer bevorstehenden Frühgeburt zwischen $28+0$ bis $36+6$ SSW eingeschlossen wurden. Die Patientinnengruppe sollte in Hinblick auf den Grenzbereich der Lebensfähigkeit möglichst wenig Bias hinsichtlich üblicher gestationsalterspezifischer Behandlungsoptionen haben und die Intervention sollte ihr zumutbar sein. Die Patientinnen wurden nach dem Zufallsprinzip so eingeteilt, dass sie gebeten wurden, entweder eine Fallvignette mit einer Überlebensrate von $60 \%$ oder eine von $30 \% \mathrm{zu}$ beantworten. Die schriftlichen Fallvignetten enthielten eine detaillierte Beschreibung einer bevorstehenden Frühgeburt bei $23+6$ SSW (60\% Überlebensrate) oder $22+6 \mathrm{SSW}$ (30\% Überlebensrate) und waren bis auf die numerischen Daten genau gleich. Die Patientinnen wurden gebeten, ihre Präferenz für ,lebenserhaltende Behandlung“, „,palliative Behandlung“ oder auch „keine Präferenz“ anzugeben und einen kurzen Fragebogen mit offenen und geschlossenen Fragen auszufüllen. Zusätzlich zu den Fallvignetten wurden sechs Variablen (Bildung, Religiosität, Fa- 
milienstand, frühere Kinder, Fruchtbarkeitsbehandlung und Alter) bezüglich ihres Einflusses auf die Präferenzen der Patientinnen identifiziert.

Insgesamt wurden 64 Patientinnen in die Analyse einbezogen. Die 60\%- oder $30 \%$-Überlebensgruppe war hinsichtlich der Präferenzraten für lebenserhaltende Behandlung im Vergleich zur palliativen Behandlung nicht unterschiedlich $(46,9 \%$ gegenüber $34,4 \%$ in der $60 \%$-Überlebensgruppe und $50,0 \%$ gegenüber $40,6 \%$ in der $30 \%$-Überlebensgruppe; Odds Ratio (OR), 0,90; $95 \%$ Confidence Interval (CI), 0,31-2,63). Nur eine Minderheit der Patientinnen konnte keine Präferenz formulieren. Die Analyse der Patientinnen, die eine Präferenz formulierten, zeigte, dass eine Einstellung, wonach das bloße Überleben mindestens genauso wichtig ist wie die Lebensqualität, mit einer Präferenz für lebenserhaltende Behandlung verbunden war (OR, 10,28; $95 \%$ CI 2,94-35,90). Zunehmendes mütterliches Alter (OR, 0,77; $95 \%$ CI, 0,61-0,98) und Kinderlosigkeit (OR, 0,12; $95 \%$ CI 0,01-0,98) waren hingegen mit einer Präferenz für Palliativmedizin assoziiert. Die Mehrheit der Patientinnen gab an, gemeinsam mit ihren Partnern entscheiden zu wollen (63 von 64 [98,4\%]), und zog es vor, von ihren Ärztinnen und Ärzten aktiv in den Entscheidungsprozess einbezogen zu werden (48 von 64 [75\%]) (Kidszun et al. 2020).

\section{Diskussion der Studienergebnisse}

\section{Reflexion der Ergebnisse aus klinischer Perspektive}

Die Verallgemeinerbarkeit der Studienergebnisse ist sicher begrenzt. Die Patientinnen der Studie mussten eine einmalige Entscheidung ohne persönliches Feedback treffen und insbesondere bleibt unklar, ob sich Betroffene in der tatsächlichen Ausnahmesituation nicht anders entschieden hätten. Zudem wurden eher gut gebildete Frauen eingeschlossen und auch nur solche mit einem guten Verständnis der deutschen Sprache. Dennoch unterstützen die Studienergebnisse bisherige Erkenntnisse von Entscheidungsfindungsprozessen in der fetalen und neonatalen Medizin. Elterliche Behandlungspräferenzen scheinen auch in dieser Studie eher von individuellen Einstellungen und Werten als von Überlegungen zu numerischen Ergebnisschätzungen herzurühren. Während in dieser Studie keine Assoziation zwischen probabilistischer Prognose und elterlicher Entscheidungspräferenz nachweisbar war, zeigte sich ein signifikanter Zusammenhang mit spezifischen Werten, hier im Speziellen mit der Einstellung zu Lebensqualität. Zu einem ähnlichen Ergebnis kam eine aktuelle Studie unter Frauen im gebärfähigen Alter. Frauen, welche die Unantastbarkeit des Lebens schätzten, autonome Entscheidungen bevorzugten, eher religiös waren und eine adäquate Gesundheitskompetenz hatten, würden häufiger eine lebenserhaltende, intensivmedizinische Behandlung wählen (McDonnell et al. 2021). Zukünftige Studien sollten vermehrt die elterlichen Haltungen und Werte zum Mittelpunkt der Betrachtung machen. Ähnlich verhält es sich mit individuellen Charakteristika der Eltern. In dieser Arbeit erschien es, als ob Mütter mit zunehmendem Alter eine immer expliziter werdende Vorstellung von ihrem erwarteten Familienleben haben. Dazu können auch Überlegungen zur Lebensqualität des zukünftigen Kindes gehören, und insbesondere ob und wie ein solches Kind mit besonderen Bedürfnissen 
in deren oft komplexere, bereits bestehende Lebensbedingungen integriert werden kann. Nicht nur die individuellen Charakteristika der Eltern, sondern auch die der betroffenen Kinder scheinen Therapieentscheidungen signifikant zu beeinflussen. So konnte bereits gezeigt werden, dass das kindliche Alter unabhängig von der Prognose einen starken Einfluss auf Behandlungspräferenzen hat (Janvier et al. 2008). Der Umstand, dass sich die niedrigere Überlebensrate nicht in einer geringeren Behandlungsrate niederschlägt, könnte auch anderen Faktoren, wie dem bereits erwähnten optimism bias geschuldet sein oder Folge von Reihenfolgeeffekten sein (McDonnell et al. 2021; Nayak et al. 2021).

Das Verständnis, welche Informationen werdende Eltern, die mit einer extremen Frühgeburt konfrontiert sind, wünschen und brauchen, ist dennoch unvollständig und bedarf weiterer, konfirmatorischer Studien und ethischer Analyse. Adäquat designte, große Studien sollten nicht nur eine repräsentative Auswahl unmittelbar betroffener Familien einschließen, sondern auch die Vielschichtigkeit des Entscheidungsprozesses adressieren.

\section{Kriterien eines guten Entscheidungsprozesses}

Der Weg zu einer gemeinsamen, ,guten“ Entscheidungsfindung ist eine fortwährende Herausforderung. Besonders im Bereich der frühen Frühgeburtlichkeit und der vorgelagerten pränatalen Beratung finden sich vergleichsweise wenige ethischnormative oder ethisch-empirische Arbeiten, welche diese Problematik adressieren. Antoine Payot hat vor einigen Jahren in einer ersten empirischen Studie zeigen können, dass der Weg der Eltern und der Ärzt*innen bereits an unterschiedlichen Orten startet und spezifische Rollen und Beratungsmuster erkennen lässt (Payot et al. 2007). Während die werdenden Eltern noch in Verarbeitung und Trauer um die Komplikationen der Schwangerschaft und die zerrissenen Pläne für die geplante Elternschaft sind (parenthood project), sind die beratenden Ärzt*innen bereits voll und ganz auf das Neugeborene konzentriert (baby project). Eltern müssen zunächst konzeptualisieren, dass das Neugeborene als eigenes Lebewesen zu betrachten ist. Günstig scheint es daher, diesen Themen in der Beratung Raum zu geben, bevor spezifische Informationen über Prognose und Zustand des Kindes gegeben werden. Bezüglich der Art der Beratung ließen sich zwei grundsätzliche Muster erkennen, wobei auffiel, dass Ärzt*innen in aller Regel ihr Muster nicht änderten. Entweder wurde versucht, so neutral wie möglich zu beraten (neutral information model), oder es wurde ein Behandlungsvorschlag gemacht, den die Eltern ablehnen oder annehmen konnten (assent model). In der Untersuchung von Payot schien es für den Beratungserfolg jedoch unerheblich zu sein, welches Modell angewandt wurde. Wenn es in der Beratung gelang, die Bedürfnisse der Eltern zu erkennen und zu besprechen, wenn die Informationen, die ihnen gegeben wurden, ihre Erwartungen erfüllte, dann gelangten sie am ehesten zu einer Entscheidung, die sie als ihre eigene wahrnahmen und von der sie überzeugt waren. Gelang dies nicht, und führte der Entscheidungsprozess zu einer einsamen Entscheidung, zu etwas als negativ Erlebtem, dann haderten die Eltern noch Monate später mit ihrer Entscheidung. Angesichts dieser Ergebnisse kommt dem Erkennen elterlicher Bedürfnisse und Werte eine entscheidende Bedeutung im Entscheidungsprozess zu. Die Kriterien, die einen 
guten Beratungs- und Entscheidungsfindungsprozess definieren können, sind in großen Teilen unklar und unzureichend evaluiert. Dies ist zuvorderst darin begründet, dass die Güte des Entscheidungsprozesses nur schwer operationalisierbar ist. Es ist denkbar, dass weniger das bisher im Fokus stehende kindliche Outcome, sondern vielmehr die Art und Weise des Beratungs-, Entscheidungs- und Unterstützungsprozesses in diese Bewertung eingeht. So könnten der Grad der Unterstützung und die Erfüllung der elterlichen Bedürfnisse als wichtige zukünftig zu prüfende Parameter einer ,guten“ Entscheidung dienen. Zudem muss bei Entscheidungen im Grenzbereich der Lebensfähigkeit das Konzept der möglicherweise verfeinert und angepasst werden, da es noch zu wenig auf Emotionen und affektive Entscheidungsfindung eingeht und kaum auf den transformativen Charakter dieser spezifischen Entscheidung achtet. Entscheidungen können als transformativ betrachtet werden, wenn sie so grundlegende Veränderungen bewirken, dass die Auswirkungen der Entscheidungen nicht vorstellbar und vorhersehbar sind (Paul 2015). Das ist bei Entscheidungen im Grenzbereich der Lebensfähigkeit sicherlich der Fall. Die vielen verschiedenen hier zu erwartenden klinischen Verläufe können den Betroffenen unvorstellbar und inkohärent erscheinen. Darüber hinaus kann die Konfrontation mit extremer Frühgeburtlichkeit für Eltern ein echtes moralisches Dilemma darstellen. Eltern finden sich hier in einer Situation wieder, die aus kindlicher Perspektive immer fatal endet. Entweder wird ihr Kind versterben oder mit deutlichen gesundheitlichen Einschränkungen überleben. Wenn jede der beiden Entscheidungen als falsch empfunden wird, besteht eine hohe Wahrscheinlichkeit, dass dies bereut wird (Tessman 2017).

\section{Entscheidungsunterstützung als Rahmenmodell der gemeinsamen Entscheidungsfindung - ,When decisions are complicated, decision making must be nuanced" (Janvier et al. 2014, S. 23)}

Behandlungsentscheidungen für Frühgeborene im Grenzbereich der Lebensfähigkeit sind komplexer Natur und stellen aufgrund der Vielzahl von Einflussfaktoren in der klinischen Praxis eine enorme Herausforderung dar. Im Folgenden wird für eine Entscheidungsunterstützung als strukturierte, ethische Orientierungshilfe argumentiert, welche als Rahmenmodell für solche Behandlungsentscheidungen dienen könnte. In manchen Gesundheitsfragen können Patient*innen informierte Entscheidungen über Behandlungen selbst und allein treffen. Entscheidungen im Grenzbereich der Lebensfähigkeit gehören sicher nicht in diese Kategorie. Entscheidungen, lebenserhaltende Maßnahmen bei Frühgeborenen nicht zu beginnen oder zu beenden, sind nicht einfach zu treffen. Kein einzelnes ethisches Prinzip ist allein als Grundlage geeignet, um ethisch verantwortungsvolles Entscheiden begründen zu können. Vielmehr ist ein prozessuales Zusammenspiel verschiedener Denkweisen und Begründungsprozesse notwendig. Die Beratung sollte ergebnisoffen, transparent und für die Beteiligten nachvollziehbar gestaltet werden. Im Rahmen eines wandlungsfähigen, gesellschaftlich normierten Wertegerüstes soll elterlichen Präferenzen Vorrang gegeben werden. In der hier vorliegenden Arbeit wurde im Wesentlichen für eine primäre Herangehensweise, die zum Ziel hat, Eltern in der Entscheidungsfindung bestmöglich zu unterstützen, argumentiert. Wesentliches Element dieser Herangehensweise 
ist die Unterstützung der Eltern bei der Ausübung ihres Rechts auf Selbstbestimmung und damit der Beförderung ihrer Entscheidungsfähigkeit. Eine solche Unterstützung sollte sich ,... auf die Förderung der Entscheidungsvoraussetzungen (wie Verständnis, Bewertungsfähigkeit und Äußerungsfähigkeit) beschränken“ (Zentrale Ethikkommission bei der Bundesärztekammer 2017, S. 418) und den Elternwillen nicht mittels Suggestion oder der Strukturierung der Entscheidungsarchitektur im Sinne eines Nudging beeinflussen. Der Begriff des Nudging wurde von den Ökonomen Richard Thaler und Cass Sunstein geprägt (Thaler und Sunstein 2008). Sie beschreiben eine Methodik, die Entscheidungen durch Anreize oder den gezielten Einsatz von Heuristiken vorhersagbar macht. Die Autoren argumentieren nicht nur, dass alle Entscheidungen, die Menschen fällen, eine nicht-rationale Komponente enthalten, und dass gewisse Umstände (die Entscheidungsarchitektur) die Entscheidungen beeinflussen, sie meinen auch, diese Umstände nutzen zu dürfen, um Entscheidungen in gewissem Umfang zu steuern. Andere Autorinnen befürworten den Ansatz, medizinische Fakten, Handlungsoptionen und Handlungskonsequenzen umfassend und möglichst objektiv und wertfrei darzustellen (Gigerenzer 2014; Blumenthal-Barby 2016; Blumenthal-Barby et al. 2016). Albisser Schleger et al. und BlumenthalBarby argumentieren im Sinne einer Sicherstellung autonomer Entscheidungen für ein analytisches Vorgehen und die bewusste Vermeidung oder Abschwächung bekannter Heuristiken (Albisser Schleger et al. 2011; Blumenthal-Barby 2016). Gerd Gigerenzer sieht insbesondere die Rolle der wohlmeinenden medizinischen Expertinnen und Experten kritisch. Diese unterlägen systematischen Verzerrungen wie Selbstschutz, mangelndem Verständnis von Gesundheitsstatistiken und Interessenkonflikten und wären je nach Ausmaß dieser Verzerrung nicht oder nur bedingt geeignet, um Entscheidungen im besten Interesse von Patientinnen und Patienten zu steuern (Gigerenzer 2015).

Die in diesem Artikel dargestellten, spezifischen Besonderheiten der Entscheidungsfindung im Grenzbereich der Lebensfähigkeit (enorme prognostische Unsicherheit, vulnerable Eltern, multiple Akteure, begrenzte zeitliche Ressourcen) erfordern ein spezifisches Verständnis einer Entscheidungsunterstützung. Dies beinhaltet im Wesentlichen, dass Eltern in dem Maße unterstützt werden, in dem sie es benötigen. Fürsorge und Wahrung der Entscheidungsautonomie stellen in diesem Zusammenhang keinen Antagonismus dar, sondern stehen in einem Verhältnis gegenseitiger Abhängigkeit (Jox 2004). Die Eltern werden bei der Wahrung ihrer Autonomie fürsorglich unterstützt. Autonomie wird hier als relational - sich in Beziehungen realisierend - verstanden.

Das vertrauenswürdige und offene Gespräch ist eine unabdingbare Voraussetzung für eine gelungene Entscheidungsunterstützung. Ob Eltern für sich selbst, für ihr Kind und ihre Familie gute Entscheidungen treffen, hängt zudem wesentlich davon $\mathrm{ab}$, ob sie die Entscheidungen als für sie konsistent erleben - also ob es gelingen kann, mit den Eltern zu einer Entscheidung zu kommen, die ihrem Erleben, ihren Werten und Ansichten entspricht. Wenn die Eltern die ultimativen Entscheider bleiben sollen, aber Schwierigkeiten haben, zu einer Entscheidung zu kommen, dann kann der Ansatz dahingehen, die Entscheidungsunterstützung aktiver zu gestalten. Möglicherweise ist es in diesen Situationen vorteilhafter, Eltern Entscheidungen zu erleichtern, als diese für sie zu treffen. Um die Entscheidungskompetenz von Eltern 
zu stärken, sollten medizinische Fakten in ihrem Umfang und ihrer Qualität individualisiert werden. Auch die Präsentation einer Standardoption, welche die Eltern wählen können, kann für diese entlastend sein. Eltern sollten Feedback zu ihren Entscheidungen, als auch zu den vorher aufkommenden Gedanken und Erwägungen, erhalten. Eltern sollten darauf hingewiesen werden, wenn und wie man sie in der Entscheidungsfindung führt. Nicht zuletzt kann eine narrative Rekonstruktion helfen, die Werte, Ansichten und Bedürfnisse der Eltern zu erfahren, und für den Entscheidungsprozess zu nutzen. Oftmals haben Eltern Schwierigkeiten diese zu äußern, oder sich ihre Werte und Einstellungen bewusst zu machen. Der ärztliche Behandlungsauftrag sollte beinhalten, sich auf Werte und Ziele des individuellen Patienten einzulassen - mehr noch: Werte und Ziele des Patienten sollten aktiv eruiert werden. Eltern bei der Entscheidungsfindung zu unterstützen, ohne in unangemessener Weise Präferenzen und Werte des Behandlungsteams einzubringen, kann als die essenzielle Schwierigkeit dieses Ansatzes in der praktischen Umsetzung angesehen werden. Der Grat zwischen Entscheidungsunterstützung und Paternalismus mit unangemessener Einmischung ist schmal (Blumenthal-Barby et al. 2016). Daher erscheint es sinnvoll, das Modell der relationalen Autonomie mit dem Prinzip der Transparenz zu ergänzen. Der Grad der Unterstützung im Entscheidungsprozess sollte offengelegt werden und die Eltern stets über alle sinnvollen therapeutischen Optionen informiert sein. Der Weg der partizipativen Entscheidungsfindung sollte weniger erkunden, was Ärzt*innen in der Situation der Eltern machen würden, sondern wie Ärzt*innen Eltern helfen können, ihre eigenen Präferenzen zu erkennen und umzusetzen.

Probabilistisches medizinisches Wissen, moralische Werte, persönliche Ziele und Präferenzen, aber auch die Art und Weise wie Wahlmöglichkeiten präsentiert werden, beeinflussen shared decision-making. Das Konzept einer Entscheidungsunterstützung versucht diese Aspekte zu vereinigen. Die Unterstützung zielt darauf ab, elterliche Entscheidungen zu ermöglichen, die im Interesse ihrer betroffenen Kinder und ihrer Familien liegen. Das Konzept basiert auf drei wesentlichen Säulen: (1) Die wissenschaftliche Basis der medizinischen Fakten, insbesondere der Grad ihrer Evidenz, einschließlich der prognostischen Unsicherheit, muss offengelegt werden. Das setzt voraus, dass das Behandlungsteam bzw. der verantwortliche Behandelnde gewillt ist, die Eltern in dem Maße und in der Zielrichtung zu unterstützen, die für diese Familie am geeignetsten erscheint, unabhängig von eigenen moralischen und medizinischen Präferenzen. (2) Werturteile sollten erkannt und wenn möglich in der Beratung sowie im Entscheidungsprozess vermieden werden. Mindestens sollten sie offen und explizit kommuniziert werden. (3) Entscheidungsunterstützung sollte zielgerichtet genutzt werden. Dies kann folgende Techniken beinhalten: (a) Anpassen von Quantität und Qualität der medizinischen Fakten (z. B. optische Entscheidungshilfen), (b) „Framing“ von Optionen (z. B. durch Anbieten oder Nichtanbieten einer Standardtherapie), (c) Feedback geben, insbesondere bezüglich der Kohärenz von Entscheidungen und (d) narrative Rekonstruktion (Ermittlung relevanter Werte, Beziehungsaufbau, prozessuale Entscheidungsfindung). Ethisch verantwortungsvolle Entscheidungsunterstützung sollte nicht aufzwingen oder überreden, sie sollte einen transparenten und strukturierten Entscheidungsansatz darstellen, welcher Eltern in einem Maße unterstützt, in dem diese es wünschen. Weitere wissenschaftliche Un- 
tersuchungen sind notwendig, um die elterlichen Bedürfnisse bei Entscheidungen in medizinischen Grauzonen näher zu charakterisieren.

\section{Fazit}

Angesichts der hier dargelegten Komplexität haben Neonatolog*innen und Ethiker*innen bereits zuvor argumentiert, dass sich Ärzt*innen bei der Beratung betroffener Eltern eher ,auf den Wald konzentrieren sollten, [und] nicht auf die Bäume“ (Janvier et al. 2012, S. 803). Die vorliegende Arbeit unterstützt das Verständnis, dass neben der Bereitstellung von klaren medizinischen Informationen viele andere Faktoren und Interventionen wertvoll für einen gemeinsam getragenen Entscheidungsprozess sind. Beratende Ärzt*innen sollten ausreichend geschult sein, um die $\mathrm{zu}$ Beratenden emotional und psychologisch unterstützen zu können (Arzuaga und Cummings 2019; Dionne-Odom und White 2021). Bei der Beratung von Eltern, die mit einer extremen Frühgeburt konfrontiert sind, sollten Ärzt*innen eine vertrauensvolle Beziehung aufbauen und sich an die Bedürfnisse der Eltern anpassen. Zu diesem Zweck sollten sie die elterlichen Werte und Überzeugungen, deren Hoffnungen und Ängste aktiv herausarbeiten (Arzuaga und Cummings 2019; Kidszun und Inthorn 2021). Ärzt*innen sollten zudem aktiv explorieren, welche Rolle die Eltern im Entscheidungsprozess einnehmen wollen und wie sie Eltern in diesem Prozess unterstützen können. Prognostische Informationen sollten verständlich dargestellt und gemeinsam mit Eltern hinsichtlich deren Aussagekraft interpretiert werden (Arzuaga und Cummings 2019). In aller Regel wünschen sich Eltern einfache Antworten: Wird mein Kind überleben? Wenn es überlebt, wie wird sein Leben sein? Wie schaffen wir es, als Familie damit zu rechtzukommen? Darauf gibt es keine einfachen, keine ausreichend genauen Antworten. Letztlich ist noch unklar, welche Art von Informationen in welcher Weise präsentiert werden sollten, damit es für Eltern am hilfreichsten ist. Wahrscheinlich müssen Ärzt*innen aber zuvorderst eine tragfähige Beziehung zu den Eltern aufbauen und möglicherweise mehr Informationen von ihnen einholen als ihnen präsentieren.

Funding Open access funding provided by University of Bern

Open Access Dieser Artikel wird unter der Creative Commons Namensnennung 4.0 International Lizenz veröffentlicht, welche die Nutzung, Vervielfältigung, Bearbeitung, Verbreitung und Wiedergabe in jeglichem Medium und Format erlaubt, sofern Sie den/die ursprünglichen Autor(en) und die Quelle ordnungsgemäß nennen, einen Link zur Creative Commons Lizenz beifügen und angeben, ob Änderungen vorgenommen wurden.

Die in diesem Artikel enthaltenen Bilder und sonstiges Drittmaterial unterliegen ebenfalls der genannten Creative Commons Lizenz, sofern sich aus der Abbildungslegende nichts anderes ergibt. Sofern das betreffende Material nicht unter der genannten Creative Commons Lizenz steht und die betreffende Handlung nicht nach gesetzlichen Vorschriften erlaubt ist, ist für die oben aufgeführten Weiterverwendungen des Materials die Einwilligung des jeweiligen Rechteinhabers einzuholen.

Weitere Details zur Lizenz entnehmen Sie bitte der Lizenzinformation auf http://creativecommons.org/ licenses/by/4.0/deed.de. 


\section{Einhaltung ethischer Richtlinien}

Interessenkonflikt A. Kidszun gibt an, dass kein Interessenkonflikt besteht.

Ethische Standards Für diesen Beitrag wurden vom Autor keine Studien an Menschen oder Tieren durchgeführt. Für die aufgeführten Studien gelten die jeweils dort angegebenen ethischen Richtlinien.

\section{Literatur}

Albisser Schleger H, Oehninger NR, Reiter-Theil S (2011) Avoiding bias in medical ethical decisionmaking. Lessons to be learnt from psychology research. Med Health Care Philos 14:155-162. https:// doi.org/10.1007/s11019-010-9263-2

American Academy of Pediatrics Committee on Fetus and Newborn, Bell EF (2007) Noninitiation or withdrawal of intensive care for high-risk newborns. Pediatrics 119:401-403. https://doi.org/10.1542/ peds.2006-3180

Antommaria AHM, Collura CA, Antiel RM, Lantos JD (2015) Two infants, same prognosis, different parental preferences. Pediatrics 135:918-923. https://doi.org/10.1542/peds.2013-4044

Arzuaga BH, Cummings CL (2019) Deliveries at extreme prematurity: outcomes, approaches, institutional variation, and uncertainty. Curr Opin Pediatr 31:182-187. https://doi.org/10.1097/MOP. 0000000000000731

Barbour JB, Rintamaki LS, Ramsey JA, Brashers DE (2012) Avoiding health information. J Health Commun 17:212-229. https://doi.org/10.1080/10810730.2011.585691

Bastek TK, Richardson DK, Zupancic JAF, Burns JP (2005) Prenatal consultation practices at the border of viability: a regional survey. Pediatrics 116:407-413. https://doi.org/10.1542/peds.2004-1427

Begenau J, Schubert C, Vogt W (2010) Die Arzt-Patient-Beziehung, 1. Aufl. Kohlhammer, Stuttgart

Berger CR, Calabrese RJ (1975) Some explorations in initial interaction and beyond: toward a developmental theory of interpersonal communication. Hum Commun Res 1:99-112. https://doi.org/10.1111/j. 1468-2958.1975.tb00258.x

Blumenthal-Barby JS (2016) Biases and heuristics in decision making and their impact on autonomy. Am J Bioeth AJOB 16:5-15. https://doi.org/10.1080/15265161.2016.1159750

Blumenthal-Barby JS, Loftis L, Cummings CL et al (2016) Should neonatologists give opinions withdrawing life-sustaining treatment? Pediatrics 138(6):e20162585. https://doi.org/10.1542/peds.20162585

Boss RD, Hutton N, Sulpar LJ et al (2008) Values parents apply to decision-making regarding delivery room resuscitation for high-risk newborns. Pediatrics 122:583-589. https://doi.org/10.1542/peds. 2007-1972

Brashers DE (2001) HIV and uncertainty: managing treatment decision making. Focus 16:5-6

Brashers DE, Neidig JL, Russell JA et al (2003) The medical, personal, and social causes of uncertainty in HIV illness. Issues Ment Health Nurs 24:497-522

Bührer C, Felderhoff-Müser U, Gembruch U et al (2020) Frühgeborene an der Grenze der Lebensfähigkeit (Entwicklungsstufe S2k, AWMF-Leitlinien-Register Nr. 024/019, Juni 2020). Z Geburtshilfe Neonatol 224:244-254. https://doi.org/10.1055/a-1230-0810

Carlsson J, Svenningsen NW (1975) Respiratory insufficiency syndrome (RIS) in preterm infants with gestational age of 32 weeks and less. Neonatal management and follow-up study. Acta Paediatr Scand 64:813-821

Costeloe K, Hennessy E, Gibson AT et al (2000) The EPICure study: outcomes to discharge from hospital for infants born at the threshold of viability. Pediatrics 106:659-671

Cummings J, Committee on Fetus and Newborn (2015) Antenatal counseling regarding resuscitation and intensive care before 25 weeks of gestation. Pediatrics 136:588-595. https://doi.org/10.1542/peds. 2015-2336

Cuttini M, Nadai M, Kaminski M et al (2000) End-of-life decisions in neonatal intensive care: physicians' self-reported practices in seven European countries. Lancet 355:2112-2118. https://doi.org/10.1016/ S0140-6736(00)02378-3

Dionne-Odom JN, White DB (2021) Reconceptualizing how to support surrogates making medical decisions for critically ill patients. JAMA. https://doi.org/10.1001/jama.2021.6445

Di Stefano LM, Wood K, Mactier H et al (2021) Viability and thresholds for treatment of extremely preterm infants: survey of UK neonatal professionals. Arch Dis Child Fetal Neonatal Ed. https://doi.org/10. 1136/archdischild-2020-321273 
Duff RS, Campbell AG (1973) Moral and ethical dilemmas in the special-care nursery. N Engl J Med 289:890-894. https://doi.org/10.1056/NEJM197310252891705

Gaucher N, Nadeau S, Barbier A et al (2016) Personalized antenatal consultations for preterm labor: responding to mothers' expectations. J Pediatr. https://doi.org/10.1016/j.jpeds.2016.08.006

Gigerenzer G (2014) Should patients listen to how doctors frame messages? BMJ 349:g7091. https://doi. org/10.1136/bmj.g7091

Gigerenzer G (2015) On the supposed evidence for libertarian paternalism. Rev Philos Psychol 6:361-383. https://doi.org/10.1007/s13164-015-0248-1

Guillen U, Kirpalani H (2018) Ethical implications of the use of decision aids for antenatal counseling at the limits of gestational viability. Semin Fetal Neonatal Med 23:25-29. https://doi.org/10.1016/j.siny. 2017.10.002

Guillén Ú, Suh S, Munson D et al (2012) Development and pretesting of a decision-aid to use when counseling parents facing imminent extreme premature delivery. J Pediatr 160:382-387. https://doi. org/10.1016/j.jpeds.2011.08.070

Guillén Ú, Mackley A, Laventhal N et al (2019) Evaluating the use of a decision aid for parents facing extremely premature delivery: a randomized trial. J Pediatr 209:52-60.e1. https://doi.org/10.1016/j. jpeds.2019.02.023

Hack M, Taylor HG, Drotar D et al (2005) Poor predictive validity of the Bayley Scales of Infant Development for cognitive function of extremely low birth weight children at school age. Pediatrics 116:333-341. https://doi.org/10.1542/peds.2005-0173

Haward MF, Janvier A (2015) An introduction to behavioural decision-making theories for paediatricians. Acta Paediatr 104:340-345. https://doi.org/10.1111/apa.12948

Haward MF, Murphy RO, Lorenz JM (2008) Message framing and perinatal decisions. Pediatrics 122:109-118. https://doi.org/10.1542/peds.2007-0620

van der Heide A, van der Maas PJ, van der Wal G et al (1997) Medical end-of-life decisions made for neonates and infants in the Netherlands. Lancet 350:251-255. https://doi.org/10.1016/S01406736(97)02315-5

Hentschel R, Lindner K, Krueger M, Reiter-Theil S (2006) Restriction of ongoing intensive care in neonates: a prospective study. Pediatrics 118:563-569. https://doi.org/10.1542/peds.2005-1615

Holsti A, Adamsson M, Serenius F et al (2016) Two-thirds of adolescents who received active perinatal care after extremely preterm birth had mild or no disabilities. Acta Paediatr 105:1288-1297. https:// doi.org/10.1111/apa.13499

Håkansson S, Farooqi A, Holmgren PA et al (2004) Proactive management promotes outcome in extremely preterm infants: a population-based comparison of two perinatal management strategies. Pediatrics 114:58-64

Jansen SJT, Otten W, Stiggelbout AM (2006) Factors affecting patients' perceptions of choice regarding adjuvant chemotherapy for breast cancer. Breast Cancer Res Treat 99:35-45. https://doi.org/10.1007/ s10549-006-9178-z

Janvier A, Leblanc I, Barrington KJ (2008) Nobody likes premies: the relative value of patients' lives. J Perinatol 28:821-826. https://doi.org/10.1038/jp.2008.103

Janvier A, Lorenz JM, Lantos JD (2012) Antenatal counselling for parents facing an extremely preterm birth: limitations of the medical evidence. Acta Paediatr 101:800-804. https://doi.org/10.1111/j.16512227.2012.02695.x

Janvier A, Barrington KJ, Aziz K et al (2014) CPS position statement for prenatal counselling before a premature birth: simple rules for complicated decisions. Paediatr Child Health 19:22-24

Jox RJ (2004) Bewusstlos, aber autonom? Ethik Med 16:401-414. https://doi.org/10.1007/s00481-0040327-9

Kidszun A, Inthorn J (2021) Providing neonatal outcome estimates as an intervention-reply. JAMA Pediatr 175:325-326. https://doi.org/10.1001/jamapediatrics.2020.5170

Kidszun A, Matheisl D, Tippmann S et al (2020) Effect of neonatal outcome estimates on decision-making preferences of mothers facing preterm birth: a randomized clinical trial. JAMA Pediatr. https://doi. org/10.1001/jamapediatrics.2020.0235

Kukora SK, Boss RD (2018) Values-based shared decision-making in the antenatal period. Semin Fetal Neonatal Med 23:17-24. https://doi.org/10.1016/j.siny.2017.09.003

Lantos JD (2018) Ethical problems in decision making in the neonatal ICU. N Engl J Med 379:1851-1860. https://doi.org/10.1056/NEJMra1801063

Lantos JD (2019) Considerations to guide treatment decisions for infants born weighing less than $400 \mathrm{~g}$. JAMA Pediatr 173:418-419. https://doi.org/10.1001/jamapediatrics.2019.0177 
Larroque B, Bréart G, Kaminski M et al (2004) Survival of very preterm infants: epipage, a population based cohort study. Arch Dis Child Fetal Neonatal Ed 89:F139-144

Lemmon ME, Huffstetler H, Barks MC et al (2019) Neurologic outcome after prematurity: perspectives of parents and clinicians. Pediatrics. https://doi.org/10.1542/peds.2018-3819

Lenz M et al (2012) Entscheidungshilfen für Patienten. Dtsch Arztebl 109(22/23):401-408

Mactier H, Bates SE, Johnston T et al (2020) Perinatal management of extreme preterm birth before 27 weeks of gestation: a framework for practice. Arch Dis Child Fetal Neonatal Ed 105:232-239. https://doi.org/10.1136/archdischild-2019-318402

Martin SC, Stone AM, Scott AM, Brashers DE (2010) Medical, personal, and social forms of uncertainty across the transplantation trajectory. Qual Health Res 20:182-196. https://doi.org/10.1177/ 1049732309356284

McDonnell S, Yan K, Kim UO et al (2021) Information order for periviable counseling: does it make a difference? J Pediatr. https://doi.org/10.1016/j.jpeds.2021.03.058

McHaffie HE, Laing IA, Parker M, McMillan J (2001) Deciding for imperilled newborns: medical authority or parental autonomy? J Med Ethics 27:104-109

Mishel MH (1988) Uncertainty in illness. Image 20:225-232

Mishel MH (1990) Reconceptualization of the uncertainty in illness theory. Image 22:256-262

Nayak B, Moon J-Y, Kim M et al (2021) Optimism bias in understanding neonatal prognoses. J Perinatol 41:445-452. https://doi.org/10.1038/s41372-020-00773-1

Partridge JC, Martinez AM, Nishida H et al (2005) International comparison of care for very low birth weight infants: parents' perceptions of counseling and decision-making. Pediatrics 116:e263-e271. https://doi.org/10.1542/peds.2004-2274

Paul LA (2015) What you can't expect when you're expecting. Res Philos 92:149-170. https://doi.org/10. 11612/resphil.2015.92.2.1

Payot A, Gendron S, Lefebvre F, Doucet H (2007) Deciding to resuscitate extremely premature babies: how do parents and neonatologists engage in the decision? Soc Sci Med 64:1487-1500. https://doi. org/10.1016/j.socscimed.2006.11.016

Peerzada JM, Richardson DK, Burns JP (2004) Delivery room decision-making at the threshold of viability. J Pediatr 145:492-498. https://doi.org/10.1016/j.jpeds.2004.06.018

Reed R, Grossman T, Askin G et al (2020) Joint periviability counseling between neonatology and obstetrics is a rare occurrence. J Perinatol. https://doi.org/10.1038/s41372-020-00796-8

Rysavy MA, Li L, Bell EF et al (2015) Between-hospital variation in treatment and outcomes in extremely preterm infants. N Engl J Med 372:1801-1811. https://doi.org/10.1056/NEJMoa1410689

Saigal S, Stoskopf BL, Feeny D et al (1999) Differences in preferences for neonatal outcomes among health care professionals, parents, and adolescents. JAMA 281:1991-1997

Saigal S, Rosenbaum PL, Feeny D et al (2000) Parental perspectives of the health status and health-related quality of life of teen-aged children who were extremely low birth weight and term controls. Pediatrics 105:569-574

Serenius F, Ewald U, Farooqi A et al (2016) Neurodevelopmental outcomes among extremely preterm infants 6.5 years after active perinatal care in Sweden. JAMA Pediatr 170:954-963. https://doi.org/ 10.1001/jamapediatrics.2016.1210

Stacey D, Légaré F, Lewis K et al (2017) Decision aids for people facing health treatment or screening decisions. Cochrane Database Syst Rev 4:CD1431. https://doi.org/10.1002/14651858.CD001431.pub5

Tessman L (2017) When doing the right thing is impossible, 1. Aufl. Oxford University Press, New York

Thaler RH, Sunstein CR (2008) Nudge: improving decisions about health, wealth and happiness. Yale University Press, New Haven

Versluys Z, de Leeuw R (1995) A Dutch report on the ethics of neonatal care. J Med Ethics 21:14-18

Wilkinson D, Verhagen E, Johansson S (2018) Thresholds for resuscitation of extremely preterm infants in the UK, Sweden, and Netherlands. Pediatrics 142:574-584. https://doi.org/10.1542/peds.2018-0478I

Zentrale Ethikkommission bei der Bundesärztekammer (2017) Entscheidungsfähigkeit und Entscheidungsassistenz in der Medizin. Jahrb Wiss Ethik 22:409-424 